\title{
Asymptotic stability estimates for some evolution problems with singular convection field
}

\author{
Fernando Farroni ${ }^{1}$
}

Received: 12 June 2020 / Accepted: 22 September 2020 / Published online: 9 October 2020

(c) The Author(s) 2020

\section{Abstract}

We establish asymptotic stability estimates for solutions to evolution problems with singular convection term. Such quantitative estimates provide a measure with respect to the time variable of the distance between the solution to a parabolic problem from the one of the its elliptic stationary counterpart.

Keywords Evolution problems · Asymptotic estimates · Convection term

\section{Mathematics Subject Classification 35K45}

\section{Introduction}

This paper concerns evolution problems whose model case reads as follows

$$
\left\{\begin{array}{l}
u_{t}-\operatorname{div}\left[M(x, t) \nabla u+A \frac{x}{|x|^{2}} u\right]=-\operatorname{div} F \quad \text { in } \Omega_{T}, \\
u=0 \quad \text { on } \partial \Omega \times(0, T), \\
u(x, 0)=u_{0}(x) \quad \text { in } \Omega,
\end{array}\right.
$$

Here and in what follows $\Omega$ denotes a regular bounded domain of $\mathbb{R}^{N}$ with $N \geq 3$, $A>0, T \in(0, \infty]$ and $\Omega_{T}$ stands for the cylinder $\Omega \times(0, T)$. With regard to the structure assumptions of the problem, we assume that $M=M(x, t): \Omega \times(0, T) \rightarrow$

The Author has been partially supported by the Gruppo Nazionale per l'Analisi Matematica, la Probabilità e le loro Applicazioni (GNAMPA) of the Istituto Nazionale di Alta Matematica (INdAM).

$凶$ Fernando Farroni

fernando.farroni@unina.it

1 Dipartimento di Matematica e Applicazioni R. Caccioppoli, Università degli Studi di Napoli Federico II, Complesso Monte S. Angelo, via Cinthia, 80126 Naples, Italy 
$\mathbb{R}^{N \times N}$ is a measurable, symmetric, matrix field satisfying the uniform bounds

$$
\lambda|\xi|^{2} \leq\langle M(x, t) \xi, \xi\rangle \leq \Lambda|\xi|^{2}
$$

for every $\xi \in \mathbb{R}^{N}$ and for a.e. $(x, t) \in \Omega \times(0, T)$ where $0<\lambda \leq \Lambda$. For the data of the problem we assume that

$$
F \in L^{2}\left(\Omega_{T}, \mathbb{R}^{N}\right) \quad \text { and } \quad u_{0} \in L^{2}(\Omega)
$$

The aim of this note is to provide a quantitative estimate related to the long time behavior of the global in time weak solution $u=u(x, t)$ of (1.1) (according to Definition 3.1 below). As an example, we wonder whether the solution $u=u(x, t)$ defined in the whole of $\Omega_{\infty}$ tends toward the one of the stationary problem

$$
\left\{\begin{array}{l}
-\Delta v-\operatorname{div}\left[A \frac{x}{|x|^{2}} v\right]=-\operatorname{div} f \quad \text { in } \Omega, \\
v=0 \quad \text { on } \partial \Omega,
\end{array}\right.
$$

as $t \rightarrow \infty$. For the data and for the structure assumptions relative to problem (1.4), we assume

$$
f \in L^{2}\left(\Omega, \mathbb{R}^{N}\right)
$$

If all the assumptions above are fulfilled, an important property for the elliptic problem (1.4) relies on the fact that that if a solution exists then it is automatically unique (see e.g. [16]). Obseve that our problem exhibits an unbounded and singular convection term if $0 \in \Omega$, because of the presence of coefficient $E_{A}(x):=A \frac{x}{|x|^{2}}$.

We introduce the following functions

$$
\begin{aligned}
K(t) & :=1+\|M(\cdot, t)-\mathbf{I}\|_{L^{\infty}(\Omega)} \\
H_{0}(t) & :=\|F(\cdot, t)-f\|_{L^{2}(\Omega)}
\end{aligned}
$$

which can be read as measures in time of the distances between the matrix $M$ and the identity $\mathbf{I}$ and $F$ and $f$ respectively. We assume that

$$
\text { there exists } t_{0} \in[0, T) \text { such that }(K-1)^{2}, H_{0}^{2} \in L^{1}\left(\left[t_{0}, T\right)\right)
$$

Finally, we set

$$
\mathcal{K}:=(K-1)^{2}\|\nabla v\|_{L^{2}(\Omega)}^{2}+H_{0}^{2}
$$

We assume that $\Omega$ contains the origin (so that the coefficient $E_{A}$ is singular) and we state our result related to problem (1.1). 
Theorem 1.1 Assume that the solutions to problems (1.1) and (1.4) exist. If $0 \in \Omega$ and if

$$
A<\frac{N-2}{4} \lambda
$$

then

$$
\|u(t)-v\|_{L^{2}(\Omega)}^{2} \leq\left\|u\left(t_{0}\right)-v\right\|_{L^{2}(\Omega)}^{2} e^{-\mu\left(t-t_{0}\right)}+C_{0} \int_{t_{0}}^{t} \mathcal{K}(s) d s
$$

for some positive constants $\mu$ and $C_{0}$. Moreover, if $T=\infty$ and $\mathcal{K} \in L^{1}\left(\left[t_{0}, \infty\right)\right)$ then

$$
\|u(t)-v\|_{L^{2}(\Omega)}^{2} \leq\left[\left\|u\left(t_{0}\right)-v\right\|_{L^{2}(\Omega)}^{2}+C_{0}\|\mathcal{K}\|_{L^{1}\left(\left[t_{0}, \infty\right)\right)}\right] e^{-\frac{\mu}{2} t}+C_{0} \int_{t / 2}^{t} \mathcal{K}(s) d s
$$

In the latter case, we have

$$
\|u(t)-v\|_{L^{2}(\Omega)}^{2} \rightarrow 0 \quad \text { as } t \rightarrow \infty
$$

Let us spend few comments on condition (1.8). First, one can observe that the time independent coefficient $E_{A}(x):=A \frac{x}{|x|^{2}}$ appearing in (1.1) actually belongs to the Marcinkiewicz space $L^{N, \infty}(\Omega)$ (we refer the reader to Sect. 2.1 for the definition and the basic properties of this function space) but does not belong to $L^{N}(\Omega)$ as long as $0 \in \Omega$. Moreover

$$
\operatorname{dist}_{L^{N, \infty}(\Omega)}\left(\left|E_{A}\right|, L^{\infty}(\Omega)\right)=A \omega_{N}^{1 / N}
$$

where $\omega_{N}$ stands for the measure of the unit ball in $\mathbb{R}^{N}$. In (1.11) the distance from $L^{\infty}$ in $L^{N, \infty}$ appears, as defined in Sect. 2.1 below. With regard to general problems of the type

$$
\left\{\begin{array}{l}
u_{t}-\operatorname{div}[M(x, t) \nabla u+E(x, t) u]=-\operatorname{div} F \quad \text { in } \Omega_{T} \\
u=0 \quad \text { on } \partial \Omega \times(0, T) \\
u(x, 0)=u_{0}(x) \quad \text { in } \Omega
\end{array}\right.
$$

the results of [9] state that one cannot expect existence of a solution (according again to Definition 3.1 below) unless we assume some uniform with respect to the time variable bound on the distance of the convective field $E$ from $L^{\infty}$ in $L^{N, \infty}$. Therefore, condition (1.8) seems quite natural in our framework in light of (1.11).

Comparison quantitative estimates between solutions of evolutionary and stationary problems as in (1.9) or (1.10) (see also (3.19) or (3.20) below) are available in [17] for equations not having lower order terms. It should be also worth to mention that recently in [10] new estimates for the behaviour at infinity of solutions to a wide class of parabolic partial differential equations (including also anisotropic type equations) have been considered.

Among all possible equations taking a form as in (1.1) we mention the following homogeneous one

$$
u_{t}-\Delta u-\operatorname{div}(E(x, t) u)=0
$$


which is known as Fokker-Planck equation. Its relevance in literature depends upon the fact that such equation describes the evolution of some Brownian motion and of some Mean Field Game. In case the convective term is bounded, many results are available in literature (see e.g. [5] and the references therein). On the other hand, in some context (see e.g. the case of the diffusion model for semiconductor devices in [6]) the boundedness of the convective field is not immediately guarantee. In addition to Definition 3.1 below, further definitions of solutions have been introduced for problem (1.1) under consideration as the renormalized solution (see e.g. [19] where the Fokker-Planck equation is coupled with some Hamilton-Jacobi-Bellman equation) and entropy solutions (see e.g. [3] where the authors do not address the existence of weak solution and obtain the existence of entropy solution assuming that $\left.E \in L^{2}\left(\Omega_{T}, \mathbb{R}^{N}\right)\right)$.

The plan of this paper is the following. In Sect. 2 we introduce the function spaces which are related to our problems and some useful results which help us in proving the asymptotic behaviour of Theorem 1.1. In Sect. 3 we will actually prove a result for a general Cauchy-Dirichlet problem, in such a way that Theorem 1.1 is a special case of this statement. The presence of the lower order term does not allow to follow [17]. We establish an estimate of decay of the super-level sets of the solution which is fundamental in order to obtain our result. Nevertheless, at the end of Sect. 3 we will underline how the assumption (1.8) comes into play for the special problem (1.1).

\section{Preliminary results}

\subsection{Lorentz spaces}

Let $\Omega$ be a bounded open subset of $\mathbb{R}^{N}$. From now on the Lebesgue measure of a measurable subset $E$ of $\mathbb{R}^{N}$ will be denoted by $|E|$. Fixed $p, q \in(1, \infty)$, the Lorentz space $L^{p, q}(\Omega)$ corresponds to the class of all measurable functions $g$ defined on $\Omega$ for which the quantity

$$
\|g\|_{p, q}=\left(p \int_{0}^{\infty}\left|\Omega_{\tau}\right|^{\frac{q}{p}} h^{q-1} d \tau\right)^{1 / q}
$$

is finite, where $\Omega_{\tau}=\{x \in \Omega:|g(x)|>\tau\}$ for any $\tau>0$. A standard feature of $\|\cdot\|_{p, q}$ relies on the fact that it is equivalent to a norm with the property that $L^{p, q}(\Omega)$ becomes a Banach space when endowed with it (we refer the reader to [18]). When $p=q$, the Lorentz space $L^{p, p}(\Omega)$ reduces to the classical Lebesgue space $L^{p}(\Omega)$. On the other hand, when $q=\infty$, the class $L^{p, \infty}(\Omega)$ corresponds to the class of all measurable functions $g$ defined on $\Omega$ for which the quantity

$$
\|g\|_{p, \infty}=\sup _{E \subset \Omega}|E|^{\frac{1}{p}-1} \int_{E}|g| d x
$$


is finite. The class $L^{p, \infty}(\Omega)$ is known as the Marcinkiewicz class and it is usually also denoted by weak $-L^{p}$. Moreover, if we set

$$
\llbracket g \rrbracket_{p, \infty}=\sup _{\tau>0} \tau\left|\Omega_{\tau}\right|^{\frac{1}{p}}
$$

it results

$$
\frac{p-1}{p^{1+\frac{1}{p}}}\|g\|_{p, \infty} \leq \llbracket g \rrbracket_{p, \infty} \leq\|g\|_{p, \infty}
$$

We refer the reader to Lemma A.2 in [2] for the proof of the latter relation.

For the Lorentz spaces the following inclusions hold

$$
L^{r}(\Omega) \subset L^{p, q}(\Omega) \subset L^{p, r}(\Omega) \subset L^{p, \infty}(\Omega) \subset L^{q}(\Omega)
$$

whenever $1 \leq q<p<r \leq \infty$. Moreover, for $1<p<\infty, 1 \leq q \leq \infty$ and $\frac{1}{p}+\frac{1}{p^{\prime}}=1, \frac{1}{q}+\frac{1}{q^{\prime}}=1$, if $f \in L^{p, q}(\Omega)$ and $g \in L^{p^{\prime}, q^{\prime}}(\Omega)$, we have the Hölder-type inequality

$$
\int_{\Omega}|f(x) g(x)| d x \leq\|f\|_{p, q}\|f g\|_{p^{\prime}, q^{\prime}}
$$

It is well known that $L^{\infty}(\Omega)$ is not a dense subspace of $L^{p, \infty}(\Omega)$. The distance to $L^{\infty}(\Omega)$ in $L^{p, \infty}(\Omega)$ is defined as

$$
\operatorname{dist}_{L^{p, \infty}(\Omega)}\left(f, L^{\infty}(\Omega)\right)=\inf _{g \in L^{\infty}(\Omega)}\|f-g\|_{L^{p, \infty}(\Omega)}
$$

We conclude this Section by recalling the Sobolev embedding theorem in the setting of Lorentz spaces in the sharp form given by [1].

Theorem 2.1 Let us assume that $1<p<N$ and $1 \leq q \leq p$. If $u \in W_{0}^{1,1}(\Omega)$ is a function whose gradient satisfies $|\nabla u| \in L^{p, q}(\Omega)$ then $u \in L^{p^{*}, q}(\Omega)$ where $p^{*}=\frac{N p}{N-p}$ is the usual Sobolev exponent and

$$
\|u\|_{p^{*}, q} \leq S_{N, p}\|\nabla u\|_{p, q}
$$

where $S_{N, p}=\omega_{N}^{-1 / N} \frac{p}{N-p}$ and $\omega_{N}$ is the measure of the unit ball in $\mathbb{R}^{N}$.

\subsection{Suitable subsets of the space $L^{\infty}\left(0, T ; L^{p, \infty}(\Omega)\right)$}

Given $T \in(0, \infty]$ and $\delta \geq 0$, we consider the subset $X_{\delta}\left(\Omega_{T}\right)$ of $L^{\infty}\left(0, T ; L^{p, \infty}(\Omega)\right)$ defined as

$$
\begin{aligned}
X_{\delta}\left(\Omega_{T}\right):= & \left\{f \in L^{\infty}\left(0, T ; L^{p, \infty}(\Omega)\right):\right. \\
& \left.\operatorname{dist}_{L^{\infty}\left(0, T ; L^{p, \infty}(\Omega)\right)}\left(f, L^{\infty}\left(0, T ; L^{\infty}(\Omega)\right)\right) \leq \delta\right\}
\end{aligned}
$$


In other words, $X_{\delta}\left(\Omega_{T}\right)$ consists of of all those functions $f \in L^{\infty}\left(0, T ; L^{p, \infty}(\Omega)\right)$ such that there exists $g \in L^{\infty}\left(0, T ; L^{\infty}(\Omega)\right)$ such that

$$
\|f-g\|_{L^{\infty}\left(0, T ; L^{p, \infty}(\Omega)\right)} \leq \delta
$$

Clearly $X_{0}\left(\Omega_{T}\right)$ is the closure of $L^{\infty}\left(\Omega_{T}\right)$ in $L^{\infty}\left(0, T ; L^{p, \infty}(\Omega)\right)$ and

$$
L^{\infty}\left(0, T ; L^{p, q}(\Omega)\right) \subset X\left(\Omega_{T}\right)
$$

for $p \leq q<\infty$.

A characterization of $X\left(\Omega_{T}\right)$ can be given in terms of the the truncation operator at level $\pm \kappa($ for $\kappa>0)$, that is

$$
T_{\kappa}(s)=\frac{s}{|s|} \min \{|s|, \kappa\}
$$

for $s \in \mathbb{R}$. The following lemma then follows (see e.g. [9]).

Lemma 2.2 For any given $\delta \geq 0, f \in X_{\delta}\left(\Omega_{T}\right)$ if and only if

$$
\lim _{\kappa \rightarrow \infty}\left\|f-T_{\kappa}(f)\right\|_{L^{\infty}\left(0, T ; L^{p, \infty}(\Omega)\right.} \leq \delta
$$

\subsection{Abstract asymptotic estimates}

An essential tool in the study of the time behaviour of our problem relies on the following result, whose proof can be found in [17].

Proposition 2.3 Let $t_{0} \geq 0$ and $T \in\left(t_{0},+\infty\right]$. Assume that $\phi=\phi(t)$ is a continuous and non negative function defined in $\left[t_{0}, T\right)$ verifying

$$
\phi\left(t_{2}\right)-\phi\left(t_{1}\right)+M \int_{t_{1}}^{t_{2}} \phi(t) d t \leq \int_{t_{1}}^{t_{2}} g(t) d t
$$

for every $t_{0} \leq t_{1}<t_{2}<T$, where $M$ is a positive constant and $g$ is a non negative function belonging to $L^{1}\left(\left[t_{0}, T\right)\right)$. Then, for every $t \geq t_{0}$ we get

$$
\phi(t) \leq \phi\left(t_{0}\right) e^{-M\left(t-t_{0}\right)}+\int_{t_{0}}^{t} g(s) d s
$$

Moreover, if $T=+\infty$ and $g$ belongs to $L^{1}\left(\left[t_{0},+\infty\right)\right)$ there exists $t_{1}>t_{0}$ such that

$$
\phi(t) \leq \Lambda e^{-\frac{M}{2} t}+\int_{t / 2}^{t} g(s) d s
$$


for every $t \geq t_{1}$, where

$$
\Lambda=\phi\left(t_{0}\right)+\int_{t_{0}}^{+\infty} g(s) d s
$$

\section{Existence and uniqueness to the a more general parabolic problem}

In this Section we consider the following evolution problem

$$
\left\{\begin{array}{l}
u_{t}-\operatorname{div}[A(x, t, \nabla u)+B(x, t, u)]=-\operatorname{div} F \quad \text { in } \Omega_{T}, \\
u=0 \quad \text { on } \partial \Omega \times(0, T), \\
u(x, 0)=u_{0}(x) \quad \text { in } \Omega,
\end{array}\right.
$$

which turns to be more general than the one in (1.1), because of the structure assumptions that we are going to describe below. Once again, $\Omega$ is a regular bounded domain of $\mathbb{R}^{N}$ with $N \geq 3, T \in(0, \infty]$ and $\Omega_{T}$ stands for the cylinder $\Omega \times(0, T)$. For the data of the problem we assume that (1.3) holds true. The vector field $A=A(x, t, \xi): \Omega_{T} \times \mathbb{R}^{N} \rightarrow \mathbb{R}^{N}$ is a Carathéodory function satisfying the following conditions

$$
\begin{aligned}
& |A(x, t, \xi)| \leq \beta|\xi|+g(x, t) \quad \text { for some } \beta>0 \text { and } g \in L^{2}\left(\Omega_{T}\right), \\
& \langle A(x, t, \xi)-A(x, t, \eta), \xi-\eta\rangle \geq \alpha|\xi-\eta|^{2} \quad \text { for some } \alpha>0
\end{aligned}
$$

for a.e. $(x, t) \in \Omega_{T}$ and for any $\xi, \eta \in \mathbb{R}^{N}$. Moreover, we assume that $B=$ $B(x, t, s): \Omega_{T} \times \mathbb{R} \rightarrow \mathbb{R}^{N}$ is a Carathéodory function satisfying the following properties

$$
\begin{aligned}
& \left|B(x, t, s)-B\left(x, t, s^{\prime}\right)\right| \leq b(x, t)\left|s-s^{\prime}\right| \\
& B(x, t, 0)=0
\end{aligned}
$$

for a.e. $x \in \Omega$, for any $t \in(0, T)$, for any $s, s^{\prime} \in \mathbb{R}$ and for some suitable measurable function $b: \Omega_{T} \rightarrow[0, \infty)$. With a slight abuse of terminology, the function $b$ in (3.4) is called convective term. Concerning the regularity of the convective term, we will assume from now on that

$$
b \in L^{\infty}\left(0, T ; L^{N, \infty}(\Omega)\right)
$$

We consider weak solutions of our problem, according to the following definition.

Definition 3.1 We say that

$$
u \in L^{\infty}\left(0, T ; L^{2}(\Omega)\right) \cap L^{2}\left(0, T ; W_{0}^{1,2}(\Omega)\right)
$$


is a weak solution to problem (3.1) if one has

$$
\begin{aligned}
& -\int_{\Omega_{T}} u \partial_{t} \varphi d x d t+\int_{\Omega_{T}}\langle A(x, t, \nabla u)+B(x, t, u), \nabla \varphi\rangle d x d t \\
& =\int_{\Omega_{T}}\langle F, \nabla \varphi\rangle d x d t+\int_{\Omega} u_{0} \varphi(0) d x
\end{aligned}
$$

for all $\varphi \in C^{\infty}\left(\Omega_{T}\right)$ with $\operatorname{supp} \varphi \subset \subset \Omega \times[0, T)$. We say that $u$ is a global weak solution if

$$
u \in L_{\mathrm{loc}}^{\infty}\left(0, \infty ; L^{2}(\Omega)\right) \cap L_{\mathrm{loc}}^{2}\left(0, \infty ; W_{0}^{1,2}(\Omega)\right)
$$

and (3.7) holds true for any given $T>0$ with $\varphi$ as before.

The main goal of the present section is to introduce suitable conditions allowing that the solution of (3.1) tends as $t \rightarrow \infty$ toward the one of the stationary problem

$$
\left\{\begin{array}{l}
\operatorname{div}[\tilde{A}(x, \nabla v)+\tilde{B}(x, v)]=\operatorname{div} f \quad \text { in } \Omega_{T}, \\
v=0 \quad \text { on } \partial \Omega
\end{array}\right.
$$

For the data relative to problem (3.8) and for the structure assumptions on the Carathéodory functions $\tilde{A}=\tilde{A}(x, \xi): \Omega \times \mathbb{R}^{N} \rightarrow \mathbb{R}^{N}$ and $\tilde{B}=\tilde{A}(x, s): \Omega \times \mathbb{R} \rightarrow$ $\mathbb{R}^{N}$, we require that

$$
\begin{aligned}
& \alpha^{\prime}|\xi-\eta|^{2} \leq\langle\tilde{A}(x, \xi)-\tilde{A}(x, \eta), \xi-\eta\rangle \\
& |\tilde{A}(x, \xi)| \leq \beta^{\prime}|\xi|+\tilde{g}(x) \quad g \in L^{2}(\Omega) \\
& \left|\tilde{B}(x, s)-\tilde{B}\left(x, s^{\prime}\right)\right| \leq \tilde{b}(x)\left|s-s^{\prime}\right| \quad \tilde{B}(x, 0)=0 \\
& \tilde{b} \in L^{N, \infty}(\Omega) \quad f \in L^{2}(\Omega)
\end{aligned}
$$

for some $0<\alpha^{\prime} \leq \beta^{\prime}<\infty$. Problem (3.1) and its stationary counterpart (3.8) have a common feature as far as existence and uniqueness of a solution are concerned. Indeed, problem (3.8) admits a unique weak solution in $W_{0}^{1,2}(\Omega)$ if

$$
\operatorname{dist}_{L^{N, \infty}(\Omega)}\left(\tilde{b}, L^{\infty}(\Omega)\right) \leq \delta
$$

for some $\delta \geq 0$ depending on the structure assumption of the problem and on $N$ (see e.g. [11,12]). It is worth mentioning that existence of a solution to (3.8) could possibly fail if $\delta$ in (3.13) is too large (see Section 4 in [12]). We also recall that in the elliptic framework a condition like (1.8) guarantees existence results (see e.g. [4]). We stress that (3.13) and (1.8) can be compared as done in the Introduction. Similarly, existence and uniqueness for problem (3.1) is obtained by assuming that the convective term $b=b(x, t)$ satisfies (3.13) uniformly with respect to the time variable, i.e.

$$
\operatorname{dist}_{L^{\infty}\left(0, T ; L^{N, \infty}(\Omega)\right)}\left(b, L^{\infty}\right) \leq \delta
$$


for some small $\delta>0$ depending only on $\alpha$ and $N$. As before, we introduce the following subset of $L^{\infty}\left(0, T ; L^{N, \infty}(\Omega)\right)$

$$
X_{\delta}\left(\Omega_{T}\right):=\left\{b \in L^{\infty}\left(0, T ; L^{N, \infty}(\Omega)\right): \text { (3.14) holds true }\right\}
$$

Let us assume from now on that some $t_{0} \geq 0$ exists such that

$$
\begin{aligned}
& \int_{\Omega}|F(x, t)-f(x)|^{2} d x \leq G_{0}(t) \\
& \int_{\Omega}|A(x, t, \nabla v)-\tilde{A}(x, \nabla v)|^{2} d x \leq G(t) \\
& \int_{\Omega}|B(x, t, v)-\tilde{B}(x, v)|^{2} d x \leq H(t)
\end{aligned}
$$

holds true for $t \geq t_{0}$ and where $G_{0}, G$ and $H$ belongs to $L^{1}\left(\left[t_{0}, \infty\right)\right)$. According to the terminology of [17], we refer to (3.16), (3.17) and (3.18) as proximity conditions.

The main result of this section reads as follows.

Theorem 3.1 Assume that both problems (3.1) and (3.8) admit solution. There exists $\delta>0$ depending only on $\alpha$ and $N$ such that, if $b \in X_{\delta}\left(\Omega_{T}\right)$ then

$$
\|u(t)-v\|_{L^{2}(\Omega)}^{2} \leq\left\|u\left(t_{0}\right)-v\right\|_{L^{2}(\Omega)}^{2} e^{-\mu\left(t-t_{0}\right)}+C_{0} \int_{t_{0}}^{t} K(s) d s
$$

for some positive constants $\mu$ and $C_{0}$ and where

$$
K(t):=\|b(t)\|_{L^{2}(\Omega)}^{2}+G_{0}(t)+G(t)+H(t)
$$

Moreover, if $u$ is a global solution of (3.1) and if $K \in L^{1}\left(\left[t_{0}, \infty\right)\right)$ then

$$
\|u(t)-v\|_{L^{2}(\Omega)}^{2} \leq\left[\left\|u\left(t_{0}\right)-v\right\|_{L^{2}(\Omega)}^{2}+C_{0}\|K\|_{L^{1}\left(\left[t_{0}, \infty\right)\right)}\right] e^{-\frac{\mu}{2} t}+C_{0} \int_{t / 2}^{t} K(s) d s
$$

We remark that stability and continuity estimates similar to the ones of Theorem 3.1 are also available in $[7-9,17]$. On the other hand, it is also worth to mention that also pointwise estimates of the spatial gradient are available in literature (see e.g. $[14,15])$.

\section{Proof of the main result}

Before we give the proof of Theorem 3.1, we focus our attention to a technical result of its own interest which describes the decay of the measure of the superlevel sets of the difference of the solutions of (3.1) and (3.8). Indeed, in the spirit of $[4,9,16]$ we prove the following lemma. 
Lemma 4.1 Assume that the solutions to problems (3.1) and (3.8) exist. Fixed $T \in$ $(0, \infty]$ and $k>0$ we have

$$
\sup _{0<t<T}|\{x \in \Omega:|u(x, t)-v(x)|>k\}| \leq C \log ^{-2}(1+k)
$$

for some constant $C>0$ which only depends on $N, \alpha,\|\nabla v\|_{L^{2}(\Omega)},\|G\|_{L^{1}(0, T)}$, $\|H\|_{L^{1}(0, T)},\left\|G_{0}\right\|_{L^{1}(0, T)}$ and $\|b\|_{L^{2}\left(\Omega_{T}\right)}$.

Proof We fix $T^{*} \in(0, T)$, we test both equations in problems (3.1) and (3.8) with $\varphi=\frac{w}{1+|w|} \chi_{\left(0, T^{*}\right)}$ where $w:=u-v$ and we subtract in order to get

$$
\begin{aligned}
\int_{0}^{T^{*}} d t \int_{\Omega}\left(u_{t}-v_{t}\right) \varphi d x+\int_{0}^{T^{*}} d t \int_{\Omega}\langle A(x, t, \nabla u)-\tilde{A}(x, \nabla v), \nabla \varphi\rangle d x \\
\quad+\int_{0}^{T^{*}} d t \int_{\Omega}\langle B(x, t, u)-\tilde{B}(x, v), \nabla \varphi\rangle d x=\int_{0}^{T^{*}} d t \int_{\Omega}\langle F-f, \nabla \varphi\rangle d x
\end{aligned}
$$

We observe that $\nabla \varphi=\frac{\nabla w}{(1+|w|)^{2}} \chi_{\left(0, T^{*}\right)}$. The first term at the left hand side of (4.2) can be estimated as follows

$$
\int_{0}^{T^{*}} d t \int_{\Omega}\left(u_{t}-v_{t}\right) \varphi d x=\int_{\Omega} d x \int_{w(x, 0)}^{w\left(x, T^{*}\right)} \frac{r}{1+|r|} d r
$$

so (4.2) is equivalent to

$$
\begin{aligned}
\int_{\Omega} d x & \int_{0}^{w\left(x, T^{*}\right)} \frac{r}{1+|r|} d r+\int_{0}^{T^{*}} d t \int_{\Omega}\langle A(x, t, \nabla u)-\tilde{A}(x, \nabla v), \nabla \varphi\rangle d x \\
& +\int_{0}^{T^{*}} d t \int_{\Omega}\langle B(x, t, u)-\tilde{B}(x, v), \nabla \varphi\rangle d x \\
& =\int_{0}^{T^{*}} d t \int_{\Omega}\langle F-f, \nabla \varphi\rangle d x+\int_{\Omega} d x \int_{0}^{w(x, 0)} \frac{r}{1+|r|} d r
\end{aligned}
$$

We observe that

$$
\int_{0}^{s} \frac{r}{1+|r|} d r=|s|-\log (1+|s|)
$$

and, since $e^{\sigma}-1-\sigma \geq \frac{1}{2} \sigma^{2}$ for any $\sigma \geq 0$, we have

$$
\int_{0}^{s} \frac{r}{1+|r|} d r \geq \frac{1}{2} \log ^{2}(1+|s|)
$$

So we have

$$
\int_{\Omega} d x \int_{0}^{w\left(x, T^{*}\right)} \frac{r}{1+|r|} d r \geq \frac{1}{2} \int_{\Omega} \log ^{2}\left(1+\left|w\left(x, T^{*}\right)\right|\right) d x
$$


From the monotonicity we have

$$
\int_{0}^{T^{*}} d t \int_{\Omega}\langle A(x, t, \nabla u)-A(x, t, \nabla v), \nabla \varphi\rangle d x \geq \alpha \int_{0}^{T^{*}} d t \int_{\Omega} \frac{|\nabla w|^{2}}{(1+|w|)^{2}} d x
$$

From (4.2) and observing that $w(\cdot, 0)=u_{0}-v$ we get

$$
\begin{aligned}
& \frac{1}{2} \int_{\Omega} \log ^{2}\left(1+\left|w\left(x, T^{*}\right)\right|\right) d x+\alpha \int_{0}^{T^{*}} d t \int_{\Omega} \frac{|\nabla w|^{2}}{(1+|w|)^{2}} d x \\
& \leq \quad \int_{0}^{T^{*}} d t \int_{\Omega}\langle\tilde{A}(x, \nabla v)-A(x, t, \nabla v), \nabla \varphi\rangle d x \\
& \quad+\int_{0}^{T^{*}} d t \int_{\Omega}\langle B(x, t, v)-B(x, t, u), \nabla \varphi\rangle d x \\
& \quad+\int_{0}^{T^{*}} d t \int_{\Omega}\langle\tilde{B}(x, v)-B(x, t, v), \nabla \varphi\rangle d x+\int_{0}^{T^{*}} d t \int_{\Omega}\langle F-f, \nabla \varphi\rangle d x \\
& \quad+\frac{1}{2}\left\|u_{0}-v\right\|_{L^{2}(\Omega)}^{2}
\end{aligned}
$$

Because of Young's inequality, for $\delta>0$ we have

$$
\begin{aligned}
& \int_{0}^{T^{*}} d t \int_{\Omega}\langle\tilde{A}(x, \nabla v)-A(x, t, \nabla v), \nabla \varphi\rangle d x \\
& \quad \leq \frac{1}{2 \delta} \int_{0}^{T^{*}} d t \int_{\Omega}|\tilde{A}(x, \nabla v)-A(x, t, \nabla v)|^{2} d x+\frac{\delta}{2} \int_{0}^{T^{*}} d t \int_{\Omega}|\nabla \varphi|^{2} d x \\
& \int_{0}^{T^{*}} d t \int_{\Omega}\langle\tilde{B}(x, v)-B(x, t, v), \nabla \varphi\rangle d x \\
& \quad \leq \frac{1}{2 \delta} \int_{0}^{T^{*}} d t \int_{\Omega}|\tilde{B}(x, v)-B(x, t, v)|^{2} d x+\frac{\delta}{2} \int_{0}^{T^{*}} d t \int_{\Omega}|\nabla \varphi|^{2} d x
\end{aligned}
$$

and also

$$
\begin{aligned}
\int_{0}^{T^{*}} d t \int_{\Omega} & \langle B(x, t, v)-B(x, t, u), \nabla \varphi\rangle d x \\
& \leq \int_{0}^{T^{*}} d t \int_{\Omega} b(x, t)|u-v||\nabla \varphi| d x \\
& \leq \int_{0}^{T^{*}} d t \int_{\Omega} b(x, t) \frac{|\nabla w|}{1+|w|} d x \\
& \leq \frac{1}{2 \delta} \int_{0}^{T^{*}} d t \int_{\Omega} b^{2}(x, t) d x+\frac{\delta}{2} \int_{0}^{T^{*}} d t \int_{\Omega} \frac{|\nabla w|^{2}}{(1+|w|)^{2}} d x
\end{aligned}
$$


Taking into account all the above estimates, the fact that $|\nabla \varphi|^{2} \leq \frac{|\nabla w|^{2}}{(1+|w|)^{2}} \chi_{\left(0, T^{*}\right)}$ and (4.4), we have

$$
\begin{aligned}
\frac{1}{2} \int_{\Omega} & \log ^{2}\left(1+\left|w\left(x, T^{*}\right)\right|\right) d x+\alpha \int_{0}^{T^{*}} d t \int_{\Omega} \frac{|\nabla w|^{2}}{(1+|w|)^{2}} d x \\
\leq & \frac{1}{2 \delta} \int_{0}^{T^{*}} d t \int_{\Omega}|\tilde{A}(x, \nabla v)-A(x, t, \nabla v)|^{2} d x \\
& +\frac{1}{2 \delta} \int_{0}^{T^{*}} d t \int_{\Omega} b^{2}(x, t) d x+\frac{1}{2}\left\|u_{0}-v\right\|_{L^{2}(\Omega)}^{2} \\
& +\frac{1}{2 \delta} \int_{0}^{T^{*}} d t \int_{\Omega}|\tilde{B}(x, v)-B(x, t, v)|^{2} d x \\
& +\frac{1}{2 \delta} \int_{0}^{T^{*}} d t \int_{\Omega}|F-f|^{2} d x+\frac{3}{2} \delta \int_{0}^{T^{*}} d t \int_{\Omega} \frac{|\nabla w|^{2}}{(1+|w|)^{2}} d x
\end{aligned}
$$

We choose $\delta=\alpha / 3$ and reabsorb at the left hand side to get

$$
\begin{aligned}
& \frac{1}{2} \int_{\Omega} \log ^{2}\left(1+\left|w\left(x, T^{*}\right)\right|\right) d x+\frac{\alpha}{2} \int_{0}^{T^{*}} d t \int_{\Omega} \frac{|\nabla w|^{2}}{(1+|w|)^{2}} d x \\
& \leq C\left[\int_{0}^{T^{*}} d t \int_{\Omega}|\tilde{A}(x, \nabla v)-A(x, t, \nabla v)|^{2} d x+\int_{0}^{T^{*}} d t \int_{\Omega} b^{2}(x, t) d x\right. \\
& \quad+\int_{0}^{T^{*}} d t \int_{\Omega}|\tilde{B}(x, v)-B(x, t, v)|^{2} d x+\int_{0}^{T^{*}} d t \int_{\Omega}|F-f|^{2} d x \\
& \left.\quad+\left\|u_{0}-v\right\|_{L^{2}(\Omega)}^{2}\right]
\end{aligned}
$$

Since $T^{*}$ can be arbitrarily chosen in $(0, T)$ and recalling conditions (3.16), (3.17) and (3.18), we immediately obtain the desired conclusion.

Proof of Theorem 3.1 Let us require that

$$
b \in X_{\delta}\left(\Omega_{\infty}\right) \quad \text { for } \delta \in\left(0, \frac{\alpha}{8 S_{N, 2}}\right)
$$

where $S_{N, 2}$ is the sharp Sobolev constant appearing in (2.2) whenever $p=2$. This means that

$$
\operatorname{dist}_{L^{\infty}\left(0, T ; L^{N, \infty}(\Omega)\right)}\left(b, L^{\infty}\right)<\frac{\alpha}{8 S_{N, 2}}
$$

and so there exists $M>0$ such that

$$
\sup _{0<t<T}\left\|b(\cdot, t)-T_{M}(b(\cdot, t))\right\|_{L^{N, \infty}(\Omega)}<\frac{\alpha}{8 S_{N, 2}}
$$


For fixed $t_{1}, t_{2} \in\left(t_{0}, \infty\right)$ with $t_{1}<t_{2}$, we use $\varphi=(u-v) \chi_{\left(t_{1}, t_{2}\right)}$ as a test function in both (3.1) and (3.8) and subtract the results obtained from this method to get

$$
\begin{aligned}
& \frac{1}{2} \int_{\Omega}|u-v|^{2}\left(t_{2}\right) d x-\frac{1}{2} \int_{\Omega}|u-v|^{2}\left(t_{1}\right) d x \\
& \quad+\int_{t_{1}}^{t_{2}} d t \int_{\Omega}\langle A(x, t, \nabla u)-A(x, t, \nabla v), \nabla u-\nabla v\rangle d x \\
& \quad+\int_{t_{1}}^{t_{2}} d t \int_{\Omega}\langle A(x, t, \nabla v)-\tilde{A}(x, \nabla v), \nabla u-\nabla v\rangle d x \\
& \quad+\int_{t_{1}}^{t_{2}} d t \int_{\Omega}\langle B(x, t, u)-B(x, t, v), \nabla u-\nabla v\rangle d x \\
& \quad+\int_{t_{1}}^{t_{2}} d t \int_{\Omega}\langle B(x, t, v)-\tilde{B}(x, v), \nabla u-\nabla v\rangle d x \\
& =\int_{t_{1}}^{t_{2}} d t \int_{\Omega}\langle F-f, \nabla u-\nabla v\rangle d x
\end{aligned}
$$

We have rearranged the terms in (4.12) so that we may argue as in the proof of Lemma 4.1 to obtain

$$
\begin{aligned}
\frac{1}{2} \int_{\Omega} \mid u & -\left.v\right|^{2}\left(t_{2}\right) d x-\frac{1}{2} \int_{\Omega}|u-v|^{2}\left(t_{1}\right) d x+\frac{\alpha}{2} \int_{t_{1}}^{t_{2}} d t \int_{\Omega}|\nabla u-\nabla v|^{2} d x \\
& \leq C \int_{t_{1}}^{t_{2}}\left(G_{0}(t)+G(t)+H(t)\right) d t+\int_{t_{1}}^{t_{2}} d t \int_{\Omega} b(x, t)|u-v||\nabla u-\nabla v| d x
\end{aligned}
$$

The only issue that matters is to estimate the latter term in (4.13). We observe that

$$
\begin{aligned}
& \int_{t_{1}}^{t_{2}} d t \int_{\Omega} b(x, t)|u-v||\nabla u-\nabla v| d x \\
& \quad \leq \int_{t_{1}}^{t_{2}} d t \int_{\Omega}\left|b(x, t)-T_{M}(b(x, t))\right| u-v|| \nabla u-\nabla v \mid d x \\
& \quad+\int_{t_{1}}^{t_{2}} d t \int_{\Omega} T_{M}(b(x, t))|u-v||\nabla u-\nabla v| d x
\end{aligned}
$$

We set $\omega_{k}(t):=\mid\{x \in \Omega:|u(x, t)-v(x)|>k \mid$. We use (2.1), Sobolev inequality (2.2) and (4.11) to get

$$
\begin{aligned}
\int_{t_{1}}^{t_{2}} d t \int_{\Omega} & b(x, t)|u-v \| \nabla u-\nabla v| d x \\
\leq & \int_{t_{1}}^{t_{2}} \int_{\Omega}\left\|b(x, t)-T_{M}(b(x, t))\right\|_{L^{N, \infty}}\|u-v\|_{L^{2^{*}, 2}(\Omega)}\|\nabla u-\nabla v\|_{L^{2}(\Omega)} d t \\
& +\int_{t_{1}}^{t_{2}} d t \int_{\Omega} T_{M}(b(x, t))|u-v \| \nabla u-\nabla v| d x
\end{aligned}
$$




$$
\begin{aligned}
& \leq \frac{\alpha}{8} \int_{t_{1}}^{t_{2}} \int_{\Omega}\|\nabla u-\nabla v\|_{L^{2}(\Omega)}^{2} d t+\int_{t_{1}}^{t_{2}} d t \int_{\Omega} T_{M}(b(x, t)) \mid u-v \| \nabla u \\
& -\nabla v \mid d x
\end{aligned}
$$

On the other hand, using again (2.1) and (2.2)

$$
\begin{aligned}
& \int_{t_{1}}^{t_{2}} d t \int_{\Omega} T_{M}(b(x, t))|u-v||\nabla u-\nabla v| d x \\
& \leq k \int_{t_{1}}^{t_{2}} d t \int_{|u-v| \leq k} T_{M}(b(x, t))|\nabla u-\nabla v| d x \\
& +M \int_{t_{1}}^{t_{2}} d t \int_{|u-v|>k}|u-v||\nabla u-\nabla v| d x \\
& \leq k \int_{t_{1}}^{t_{2}} d t \int_{|u-v| \leq k} T_{M}(b(x, t))|\nabla u-\nabla v| d x \\
& +M \int_{t_{1}}^{t_{2}} d t \int_{|u-v|>k}|u-v||\nabla u-\nabla v| d x \\
& \leq k \int_{t_{1}}^{t_{2}} d t \int_{|u-v| \leq k} T_{M}(b(x, t))|\nabla u-\nabla v| d x \\
& +M S_{N, 2} \int_{t_{1}}^{t_{2}} \omega_{k}(t)^{\frac{1}{N}}\|\nabla u-\nabla v\|_{L^{2}(\Omega)}^{2} d t
\end{aligned}
$$

Due to Lemma 4.1, we have $\omega_{k}(t) \rightarrow 0$ as $k \rightarrow \infty$ uniformly w.r.t. $t \in(0, T)$. So, if we choose $k$ sufficiently large to have

$$
\begin{aligned}
& \int_{t_{1}}^{t_{2}} d t \int_{\Omega} T_{M}(b(x, t))|u-v||\nabla u-\nabla v| d x \\
& \quad \leq k \int_{t_{1}}^{t_{2}} d t \int_{|u-v| \leq k} T_{M}(b(x, t))|\nabla u-\nabla v| d x+\frac{\alpha}{16} \int_{t_{1}}^{t_{2}}\|\nabla u-\nabla v\|_{L^{2}(\Omega)}^{2} d t
\end{aligned}
$$

Then, using Young's inequality we have

$$
\begin{aligned}
& \int_{t_{1}}^{t_{2}} d t \int_{\Omega} T_{M}(b(x, t))|u-v \| \nabla u-\nabla v| d x \\
& \quad \leq C \int_{t_{1}}^{t_{2}}\|b(t)\|_{L^{2}(\Omega)}^{2} d t+\frac{\alpha}{8} \int_{t_{1}}^{t_{2}}\|\nabla u-\nabla v\|_{L^{2}(\Omega)}^{2} d t
\end{aligned}
$$

Inserting (4.18) in (4.15) we have

$$
\begin{aligned}
\int_{t_{1}}^{t_{2}} d t \int_{\Omega} b(x, t)|u-v \| \nabla u-\nabla v| d x \leq & \frac{\alpha}{4} \int_{t_{1}}^{t_{2}} \int_{\Omega}\|\nabla u-\nabla v\|_{L^{2}(\Omega)}^{2} d t \\
& +C \int_{t_{1}}^{t_{2}}\|b(t)\|_{L^{2}(\Omega)}^{2} d t
\end{aligned}
$$


Taking into account (4.13), we reabsorb by the left hand side and then we may find some constants $c_{1}$ and $c_{2}$ such that

$$
\frac{1}{2}\left\|w\left(t_{2}\right)\right\|_{2}^{2}-\frac{1}{2}\left\|w\left(t_{1}\right)\right\|_{2}^{2}+c_{1} \int_{t_{1}}^{t_{2}}\|\nabla w\|_{2}^{2} d t \leq c_{2} \int_{t_{1}}^{t_{2}} K(t) d t
$$

where $w:=u-v$. Finally, by means of Poincaré inequality, we have

$$
\frac{1}{2}\left\|w\left(t_{2}\right)\right\|_{2}^{2}-\frac{1}{2}\left\|w\left(t_{1}\right)\right\|_{2}^{2}+c_{3} \int_{t_{1}}^{t_{2}}\|w\|_{2}^{2} d t \leq c_{2} \int_{t_{1}}^{t_{2}} K(t) d t
$$

Thus, we may apply Proposition 2.3 to the function

$$
\phi(t):=\int_{t_{0}}^{t}\|w(x, \tau)\|_{L^{2}(\Omega)}^{2} d \tau
$$

and estimate (4.21) immediately yelds the desired result.

Proof of Theorem 1.1 With respect to the proof of previous Theorem 3.1, we perform a different argument to estimate of the term

$$
\int_{t_{1}}^{t_{2}} d t \int_{\Omega}\langle B(x, t, v)-B(x, t, u), \nabla \varphi\rangle d x
$$

appearing in (4.12). Indeed, in this case we have $B(x, t, u)=A \frac{x}{|x|^{2}} u$ and so the latter term can be estimated

$$
A \int_{t_{1}}^{t_{2}} d t \int_{\Omega}\left\langle\frac{x}{|x|^{2}} v-\frac{x}{|x|^{2}} u, \nabla \varphi\right\rangle d x \leq A \int_{t_{1}}^{t_{2}}\left\|\frac{u-v}{|x|}\right\|_{L^{2}(\Omega)}\|\nabla u-\nabla v\|_{L^{2}(\Omega)} d t
$$

Now, we make use of the classical Hardy inequality in its sharp form (see e.g. Lemma 17.1 in [20] for an elementary proof)

$$
\left(\frac{N-2}{2}\right)^{2} \int_{\Omega} \frac{U^{2}}{|x|^{2}} d x \leq \int_{\Omega}|\nabla U|^{2} d x \quad \text { for all } U \in W_{0}^{1,2}(\Omega)
$$

to get

$$
A \int_{t_{1}}^{t_{2}} d t \int_{\Omega}\left\langle\frac{x}{|x|^{2}} v-\frac{x}{|x|^{2}} u, \nabla \varphi\right\rangle d x \leq \frac{2 A}{N-2} \int_{t_{1}}^{t_{2}}\|\nabla u-\nabla v\|_{L^{2}(\Omega)}^{2} d t
$$

The latter term can be reabsorbed by the left hand side because of condition (1.8), while the rest of the proof goes without changes. 
Funding Open access funding provided by Università degli Studi di Napoli Federico II within the CRUICARE Agreement.

\section{Compliance with ethical standards}

Conflict of interest On behalf of all authors, the corresponding author states that there is no conflict of interest.

Open Access This article is licensed under a Creative Commons Attribution 4.0 International License, which permits use, sharing, adaptation, distribution and reproduction in any medium or format, as long as you give appropriate credit to the original author(s) and the source, provide a link to the Creative Commons licence, and indicate if changes were made. The images or other third party material in this article are included in the article's Creative Commons licence, unless indicated otherwise in a credit line to the material. If material is not included in the article's Creative Commons licence and your intended use is not permitted by statutory regulation or exceeds the permitted use, you will need to obtain permission directly from the copyright holder. To view a copy of this licence, visit http://creativecommons.org/licenses/by/4.0/.

\section{References}

1. Alvino, A.: Sulla disuguaglianza di Sobolev in spazi di Lorentz. Bollettino della Unione Matematica Italiana A 5(14), 148-156 (1977)

2. Bénilan, P., Brezis, H., Crandall, M.: A semilinear equation in $L^{1}\left(\mathbb{R}^{N}\right)$. Ann. Scuola Norm. Sup. Pisa Cl. Sci. (4) 2(4), 523-555 (1975)

3. Boccardo, L., Orsina, L., Porretta, A.: Some noncoercive parabolic equations with lower order terms in divergence form, Dedicated to Philippe Bénilan. J. Evol. Equ. 3(3), 407-418 (2003)

4. Boccardo, L.: Dirichlet problems with singular convection terms and applications. J. Differ. Equ. 258(7), 2290-2314 (2015)

5. Cardaliaguet, P., Lasry, J.-M., Lions, P.-L., Porretta, A.: Long time average of mean field games. Netw. Heterog. Media 7(2), 279-301 (2012)

6. Fang, W., Ito, K.: Weak solutions for diffusion-convection equations. Appl. Math. Lett. 13(3), 69-75 (2000)

7. Farroni, F., Greco, L., Moscariello, G.: Stability for $p$-Laplace type equation in a borderline case. Nonlinear Anal. 116, 100-111 (2015)

8. Farroni, F., Greco, L., Moscariello, G.: Estimates for $p$-Laplace type equation in a limiting case. Atti Accad. Naz. Lincei Rend. Lincei Mat. Appl. 25(4), 445-448 (2014)

9. Farroni, F., Moscariello, G.: A nonlinear parabolic equation with drift term. Nonlinear Anal. B 177, 397-412 (2018)

10. Frankowska, H., Moscariello, G.: Long-Time behaviour of solutions to an evolution PDE with nonstandard growth. Adv. Calc. Variat. (2020). https://doi.org/10.1515/acv-2019-0061

11. Giannetti, F., Greco, L., Moscariello, G.: Linear elliptic equations with lower-order terms. Differ. Integ. Equ. 26(5-6), 623-638 (2013)

12. Greco, L., Moscariello, G., Zecca, G.: Very weak solutions to elliptic equations with singular convection term. J. Math. Anal. Appl. 457(2), 1376-1387 (2018)

13. Greco, L., Moscariello, G., Zecca, G.: An obstacle problem for noncoercive operators. Abst. Appl. Anal. Art. ID 890289 (2015)

14. Kuusi, T., Mingione, G.: The Wolff gradient bound for degenerate parabolic equations. J. Eur. Math. Soc. (JEMS) 16(4), 835-892 (2014)

15. Kuusi, T., Mingione, G.: Riesz potentials and nonlinear parabolic equations. Arch. Ration. Mech. Anal. 212(3), 727-780 (2014)

16. Moscariello, G.: Existence and uniqueness for elliptic equations with lower-order terms. Adv. Calc. Var. 4(4), 421-444 (2011)

17. Moscariello, G., Porzio, M.M.: Quantitative asymptotic estimates for evolution problems. Nonlinear Anal. 154, 225-240 (2017)

18. O’Neil, R.: Convolution operators and $L(p, q)$ spaces. Duke Math. J. 30, 129-142 (1963) 
19. Porretta, A.: Weak solutions to Fokker-Planck equations and mean field games. Arch. Ration. Mech. Anal. 216(1), 1-62 (2015)

20. Tartar, L.: An Introduction to Sobolev Spaces and Interpolation Spaces. Lect. Notes Unione Mat. Italiana 3. Springer, Berlin(2007)

Publisher's Note Springer Nature remains neutral with regard to jurisdictional claims in published maps and institutional affiliations. 